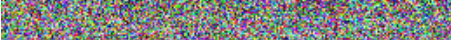

This information is current as of April 26, 2023.

\title{
Susceptibility-Weighted Imaging in the Diagnosis of Early Basal
} Ganglia Germinoma

X. Lou, L. Ma, F.-L. Wang, Z.-P. Tang, H. Huang, Y.-Q. Cai and E.-H.-C. Wong

AJNR Am J Neuroradiol 2009, 30 (9) 1694-1699

doi: https://doi.org/10.3174/ajnr.A1696

http://www.ajnr.org/content/30/9/1694 


\section{ORIGINAL RESEARCH}

\author{
X. Lou \\ L. Ma \\ F.-L. Wang \\ Z.-P. Tang \\ $H$. Huang \\ Y.-O. Cai \\ E.-H.-C. Wong
}

\section{Susceptibility-Weighted Imaging in the Diagnosis of Early Basal Ganglia Germinoma}

BACKGROUND AND PURPOSE: Germinomas originating from the basal ganglia (BG) are rare. Early diagnosis is important for favorable prognosis, but it is difficult due to the slow clinical course and subtle changes on neuroimaging. The purpose of this study was to evaluate the usefulness of susceptibility-weighted imaging (SWI) in the diagnosis of early BG germinoma.

MATERIALS AND METHODS: From 2006 to 2008, 6 BG germinomas were diagnosed in children at our institution by pathology. Conventional MR imaging and SWI were available in all cases. Clinical, neuroradiologic, and follow-up features were retrospectively studied.

RESULTS: Three cases were classified as early BG germinomas. Conventional MR imaging demonstrated that the tumor size was $<10 \mathrm{~mm}$ in the largest diameter. The tumors were invisible or showed slight hyperintensity on T1-weighted images (T1WI) and patchy slight hyperintensity on T2-weighted images (T2WI) without mass effect or enhancement. On SWI, the tumors appeared as obvious hypointensity in the globus pallidus and putamen, and the size was larger than that on conventional $\mathrm{T} 1 \mathrm{WI}$ and T2WI. The other 3 cases with tumor size $>10 \mathrm{~mm}$ in largest diameter were classified as late BG germinomas, with tumor necrosis, fluid-fluid levels, and perifocal edema, including 1 case with subependymal spread. On SWI, only the solid portion of the tumors showed hypointensity. No recurrence was noted on follow-up.

CONCLUSIONS: SWI appears to be more sensitive in detecting early BG germinomas than conventional MR imaging. This capability may prove to be useful in future attempts to characterize early BG germinomas.

$\mathbf{G}^{\mathrm{e}}$ erminomas situated in the basal ganglia (BG) are rare. ${ }^{1}$ Most of those reported in articles have been from East Asia. ${ }^{1-3}$ MR imaging has played an important role in the diagnosis of BG germinoma, even when the serum $\alpha$-fetoprotein (AFP) and human chorionic gonadotropin (HCG) levels are normal. ${ }^{4-6}$ Compared with the germinomas in other locations, the typical image finding of BG germinoma is the relatively large tumor size with a mild space-occupying effect ${ }^{7,8}$; and cystic formation, calcification, and intratumoral bleeding are commonly seen. ${ }^{1}$

Because germinomas are highly sensitive to radiation therapy (RT), which can achieve a curable outcome without surgical intervention, ${ }^{9}$ early diagnosis is important for treatment and prognosis. Conventional MR imaging, including T1weighted images (T1WI), T2-weighted images (T2WI), and postgadolinium T1WI sequences, has been a valuable tool in the diagnosis of BG germinomas. However, early diagnosis is still difficult due to the typical slow progression, lack of spaceoccupying effect, and subtle signal-intensity changes in these tumors. $^{3}$

Pathology has demonstrated that hemorrhage and iron deposition, which may occur in germinomas even at the early

\section{Received March 1, 2009; accepted after revision April 28}

From the Departments of Radiology (X.L., L.M., H.H., Y.-Q.C.) and Pathology (F.-L.W.), People's Liberation Army General Hospital, Beijing, China; Department of Neurology (Z.-P.T.), Tongji Hospital, Tongji Medical College, Huazhong University of Science and Technology, Wuhan, China; and Department of Medicine and Therapeutics (E.-H.-C.W.), Chinese University of Hong Kong, Hong Kong, China.

Please address correspondence to L. Ma, MD, Department of Radiology, People's Liberation Army General Hospital, No. 28 Fuxing Rd, Beijing 100853, China; e-mail: cjr.malin@ vip.163.com

Indicates open access to non-subscribers at www.ajnr.org

DOI 10.3174/ajnr.A1696 stage, may be helpful for early tumor detection. ${ }^{10-13}$ Susceptibility-weighted imaging (SWI) has exquisite sensitivity to the venous vasculature and blood products (such as those that deposit after hemorrhage) and biologic metal accumulation. ${ }^{14}$ Therefore, in theory, the evolution of hemorrhage and iron deposition in the early stage of BG germinomas can be tracked by using SWI.

We report a series of 6 cases of BG germinomas. Conventional MR imaging and SWI were available in all cases. The goal of the study was to evaluate the usefulness of conventional MR imaging and SWI in the diagnosis of early BG germinomas.

\section{Materials and Methods}

\section{Patients}

Between June 2006 and December 2008, 6 germinomas arising from the BG and thalamus were diagnosed in teenagers at our institution on the basis of pathologic findings. Age at diagnosis, sex, duration of symptoms, clinical presentations, AFP and HCG levels, tumor locations, and MR imaging findings were recorded and are summarized in Table 1 . The study was conducted within the guidelines from the institutional review board, and informed consent was obtained in all cases.

\section{Conventional MR Imaging and SWI}

All the MR images were acquired on a 3T whole-body scanner (Signa, TwinSpeed 3T; GE Healthcare, Milwaukee, Wis) with an 8-channel head coil. The protocols and parameters of conventional MR imaging were as follows: axial and coronal periodically rotated overlapping parallel lines with enhanced reconstruction fast spin-echo T2WI (TR/ $\mathrm{TE}=5000 / 113.7 \mathrm{~ms})$; axial and sagittal fluid-attenuated inversion recovery T1WI $(\mathrm{TR} / \mathrm{TE} / \mathrm{TI}=2000 / 6.9 / 860 \mathrm{~ms})$; and postcontrast T1WI (axial, coronal, and sagittal planes) (contrast agent: 0.1- 
Table 1: Clinical and radiologic features of BG germinomas

\begin{tabular}{|c|c|c|c|c|c|c|c|c|c|c|c|c|c|}
\hline $\begin{array}{l}\text { Case } \\
\text { No. }\end{array}$ & $\begin{array}{l}\text { Age } \\
\text { (yr) }\end{array}$ & Sex & $\begin{array}{l}\text { Symptom } \\
\text { Duration } \\
\text { (mo) }\end{array}$ & $\begin{array}{l}\text { Weakness } \\
\text { of Limbs }\end{array}$ & $\begin{array}{l}\text { Diabetes } \\
\text { Insipidus }\end{array}$ & $\begin{array}{l}\text { Increased } \\
\text { ICP }\end{array}$ & Diagnosis & $\begin{array}{c}\text { Tumor } \\
\text { Marker } \\
\text { (AFP, } \\
\text { HCG) }\end{array}$ & $\begin{array}{l}\text { Tumor } \\
\text { Location }\end{array}$ & T1WI & T2WI & $\begin{array}{c}\text { Contrast } \\
\text { Enhancement }\end{array}$ & SWI \\
\hline 1 & 14 & $\mathrm{M}$ & 2 & $Y$ & $\mathrm{~N}$ & $\mathrm{~N}$ & $\mathrm{HP}$ & Neg & $\mathrm{BG} / \mathrm{TH}$ & Slight hyper & Hyper & $\mathrm{N}$ & Нypo \\
\hline 2 & 16 & $M$ & 3 & Y & Y & N & $\mathrm{HP}$ & Neg & $\mathrm{BG}$ & Iso & Hyper & $\mathrm{N}$ & Нуро \\
\hline 3 & 14 & $M$ & 6 & Y & N & Y & $\mathrm{HP}$ & $\mathrm{Neg}$ & $\mathrm{BG}$ & Hetero/hyper & Hetero & Y (hetero) & Hetero/Hypo \\
\hline 4 & 12 & $M$ & 16 & Y & $\mathrm{N}$ & $Y$ & $\mathrm{HP}$ & $\mathrm{N} / \mathrm{A}$ & $\mathrm{BG} / \mathrm{TH}$ & Hetero/hyper & Hetero & $Y$ (hetero) & Hetero/Hypo \\
\hline 5 & 13 & $M$ & 8 & Y & Y & N & $\mathrm{HP}$ & Neg & $\mathrm{BG} / \mathrm{TH}$ & Hetero & Hetero & Y (hetero) & Hetero/Hypo \\
\hline 6 & 15 & M & 2 & Y & $\mathrm{N}$ & $\mathrm{N}$ & $\mathrm{HP}$ & $\mathrm{Neg}$ & $\mathrm{BG}$ & Iso & Hyper & $\mathrm{N}$ & Нуро \\
\hline
\end{tabular}

Note:-Y indicates yes; N, no; ICP, intracranial pressure; HP, histologically proven; AFP, $\alpha$-fetoprotein; HCG, human chorionic gonadotropin; N/A, not available; Neg, negative; BG, basal ganglia; TH, thalamus; Hetero, heterogeneous; Hyper, hyperintense; Hypo, hypointense; Iso, isointense; SWI, susceptibility-weighted imaging; T1WI, T1-weighted imaging; T2WI T2-weighted imaging.

$\mathrm{mmol} / \mathrm{kg}$ gadopentetate dimeglumine, Magnevist; Bayer Schering Pharma, Berlin, Germany) with a section thickness/gap $=5 / 0 \mathrm{~mm}$ for axial and coronal planes, $3 / 0 \mathrm{~mm}$ for sagittal plane; FOV $=24 \mathrm{~cm} \times$ $24 \mathrm{~cm} ; \mathrm{NEX}=2$.

SWI was performed before contrast agent administration. The SWI sequence was a fully velocity-compensated (gradient moment nulling in all 3 orthogonal directions) 3D gradient-echo sequence with the following typical parameters: $\mathrm{TR} / \mathrm{TE}=32 / 19 \mathrm{~ms}, \mathrm{FA}=20^{\circ}$, section thickness $=2 \mathrm{~mm}$, FOV $=24 \times 24 \mathrm{~cm}$, matrix $=448 \times 384$, $\mathrm{NEX}=0.75$. The total acquisition time was from 5 to 6 minutes, depending on the spatial ratio and the number of sections.

We postprocessed phase-sensitive images by using a high-pass filter and then converted the images into negative phase masks, which we then multiplied 4 times into the corresponding magnitude images by research software (FuncTool 2 software, GE Healthcare) on the workstation ADW4.3 (Sun Microsystems, Santa Clara, Calif).

Follow-up conventional MR imaging was performed in all cases, and follow-up SWI was performed in 3 cases (cases 2, 5, and 6). The patients were followed up at a median of 12 months (range, 1-26 months). All MR images were reviewed by 2 radiologists (X.L., L.M.), and the imaging features, including tumor location, size, shape, solid and cystic components, mass effect, perifocal edema, and enhancement pattern, were analyzed.

In this study, we classified BG germinoma into early and late subtypes, according to size and radiologic appearance. Early BG germinoma was defined as tumors $<10 \mathrm{~mm}$ in largest diameter with no cystic changes, necrosis, or hemorrhage on conventional MR imaging; late BG germinoma was defined as tumors $>10 \mathrm{~mm}$ in largest diameter with cystic changes, necrosis, or hemorrhage on conventional MR imaging.

Region-of-interest measurements were made in the lesions and in the tissues adjacent to the lesions, the SD of the background noise was also measured, and then the contrast-to-noise ratio (CNR) was calculated on T1WI, T2WI, and SWI, respectively. The CNR was defined as the algebraic difference of the normalized signal intensity (SI) between the lesion and adjacent brain tissue divided by the SD of background noise $\left(\mathrm{CNR}=\mathrm{SI}_{\text {lesion }}-\mathrm{SI}_{\text {adjacent brain tissue }} / \mathrm{SD}_{\text {background noise }}\right)$.

\section{Pathologic Analysis}

Specimens from a stereotactic biopsy in 3 cases and a surgical resection in the other 3 cases were fixed in formalin and paraffin-embedded. In each case, pathologic examinations included both routine hematoxylineosin (HE) staining and immunohistochemical techniques.

\section{Table 2: Measurement of tumor size on T1WI, T2WI, and SWI}

\begin{tabular}{lccc}
\hline Case No. & T1WI $(\mathrm{mm})$ & T2WI $(\mathrm{mm})$ & SWI $(\mathrm{mm})$ \\
\hline 1 & 4 & 7 & 18 \\
2 & N/A & 8 & 21 \\
3 & 38 & 38 & 32 \\
4 & 45 & 45 & 35 \\
5 & 15 & 15 & 12 \\
6 & N/A & 8 & 20 \\
\hline
\end{tabular}

\section{Results}

\section{Clinical Features}

All 6 cases were included in the analysis (Table 1). All patients were male, and their ages ranged from 12 to 16 years (mean age, 14 years). Limb weakness was revealed in all patients; headache and vomiting (increased intracranial pressure), in 2 (cases 3 and 4); and diabetes insipidus, in 2 (cases 2 and 5). On average, the patients had symptoms ranging from 2 to 16 months (mean time, 6 months) before they came to the clinic. Five patients (cases 1, 2, 3, 5, and 6) underwent serum and CSF testing for AFP and HCG. The results were all negative, and no neoplastic germ cell was found in the CSF of these patients. RT was performed in 3 patients (cases 1, 2, and 6) after biopsy, and subtotal resection followed by RT was performed in 3 other patients (cases 3, 4, and 5).

\section{Neuroimaging}

Three patients (cases 1, 2, and 6) were classified as having early BG germinoma in this study. On conventional MR imaging, the tumor sizes (largest diameter) were $<10 \mathrm{~mm}$. On T1WI, the tumor showed slight hyperintensity in case 1 but was not visible in cases 2 and 6. On T2WI, all tumors demonstrated patchy slight hyperintensity and no mass effect. Three early BG germinomas showed no contrast enhancement after gadolinium administration. On SWI, the tumors appeared as obvious hypointensity in the globus pallidus and putamen, and their size was larger than that on conventional T1WI and T2WI (Table 2).

In case 1, thalamus involvement could be seen on SWI but was uncertain on conventional MR imaging. Because MR imaging findings, clinical symptoms, and laboratory results were not typical for germinoma in case 1 at the time of initial MR imaging, follow-up was suggested. This patient underwent the second MR examination 66 days later, and the lesion turned out to be larger than before and demonstrated evident short 

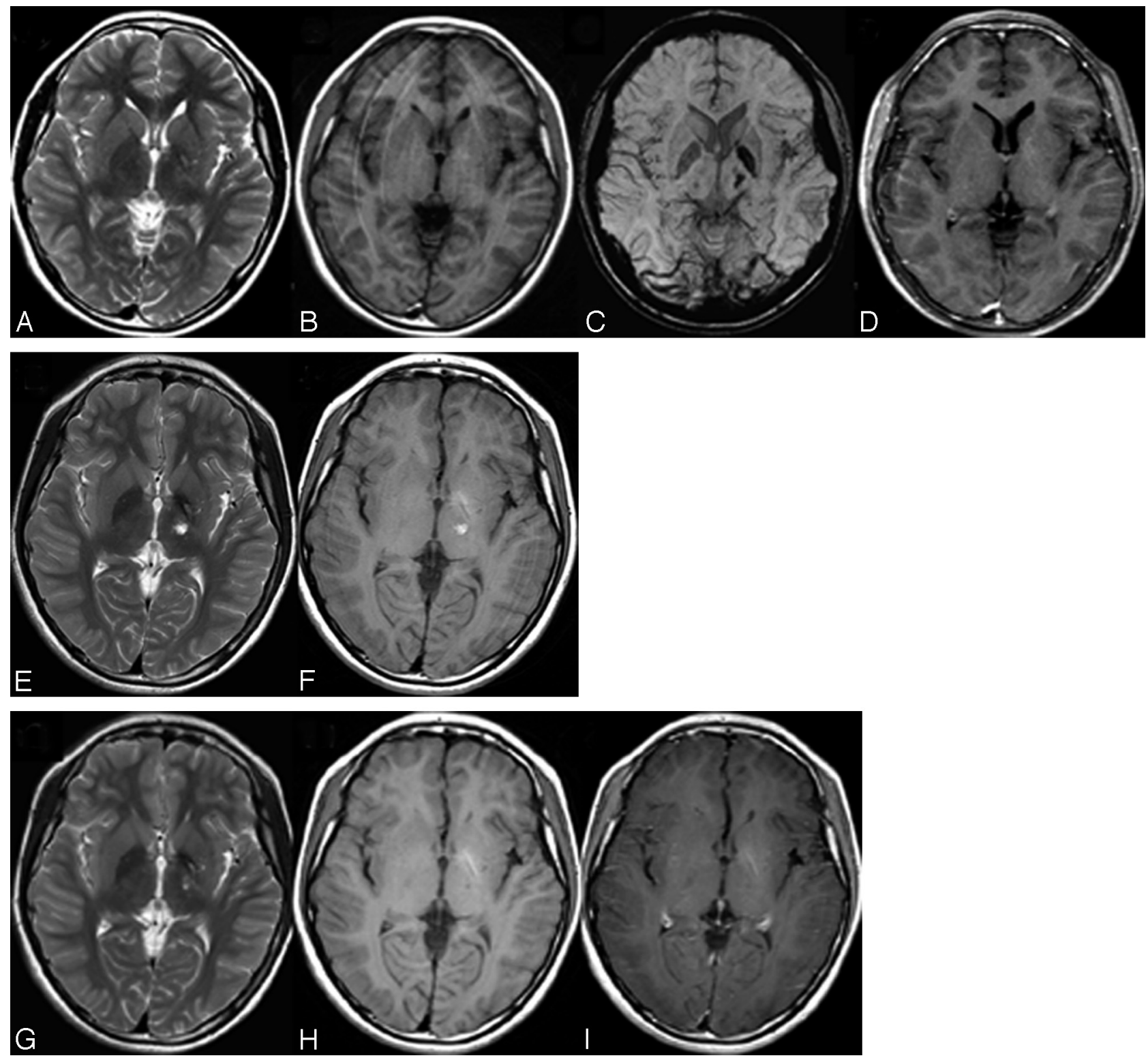

Fig 1. MR imaging of case 1. A, Axial T2WI shows patchy hyperintensity at the left BG and the thalamus. $B$, T1WI shows slight hyperintensity at the left BG without obvious mass effect. $C$, SWI shows obvious hypointensity at the left posterior limb of internal capsule, globus pallidus, and thalamus. $D$, Postcontrast T1WI shows no enhancement. $E$ and $F$, Repeat MR imaging 66 days later shows increased tumor size and hyperintensity on both T2WI $(E)$ and T1WI (F), indicating intratumoral bleeding. $G-I$, Twenty-seven days after RT, MR images show shrinkage of the tumor on T2WI $(G)$, T1WI $(H)$, and postcontrast T1WI (I). Follow-up SWI was not performed in this case.

T1 signal intensity, indicating intratumoral bleeding. RT was performed after pathologic confirmation of BG germinoma from biopsies in cases 1, 2, and 6. Follow-up MR images in case 1 revealed that the lesion had shrunk (Fig 1) 27 days after RT. Follow-up MR images in case 2 revealed that the lesion was smaller than before, with slight ringlike enhancement (Fig 2) 27 days after RT. One hundred sixty-seven days after RT, follow-up MR images in case 6 revealed that the lesion was smaller than before, with subtle enhancement.

Three patients (cases 3, 4, and 5) were classified as having late BG germinoma in this study. On conventional MR imaging, the size of the tumors in cases 3, 4, and 5 ranged from 15 to $45 \mathrm{~mm}$. The tumors were predominantly composed of solid components with fluid-fluid levels in 2 patients (cases 3 and 4 ), and the tumor was initially solid in 1 patient (case 5). On both T1WI and T2WI, the signal intensity of the tumors was heterogeneous with perifocal edema. The thalamus was involved in cases 4 and 5. Gadolinium injection induced heterogeneous enhancement of the solid portion of the tumor in all 3 cases, and subependymal spread was detected in 1 case (case 4) (Fig 3). Smaller hypointense tumor size in the BG and thalamus was demonstrated on SWI than on conventional MR images (Table 2), whereas perifocal edema and subependymal spread were not noted on SWI. Subtotal resection was then performed in these 3 late cases.

In cases 2 and 5, diabetes insipidus was noted as one of the symptoms, and the normal high signal intensity in the posterior lobe of the pituitary gland did disappear on initial sagittal T1WI. However, no mass or abnormal enhancement could be revealed in the hypothalamus or infundibular region. Follow-up MR imaging did not demonstrate new lesions in those regions, and diabetes insipidus also resolved. 

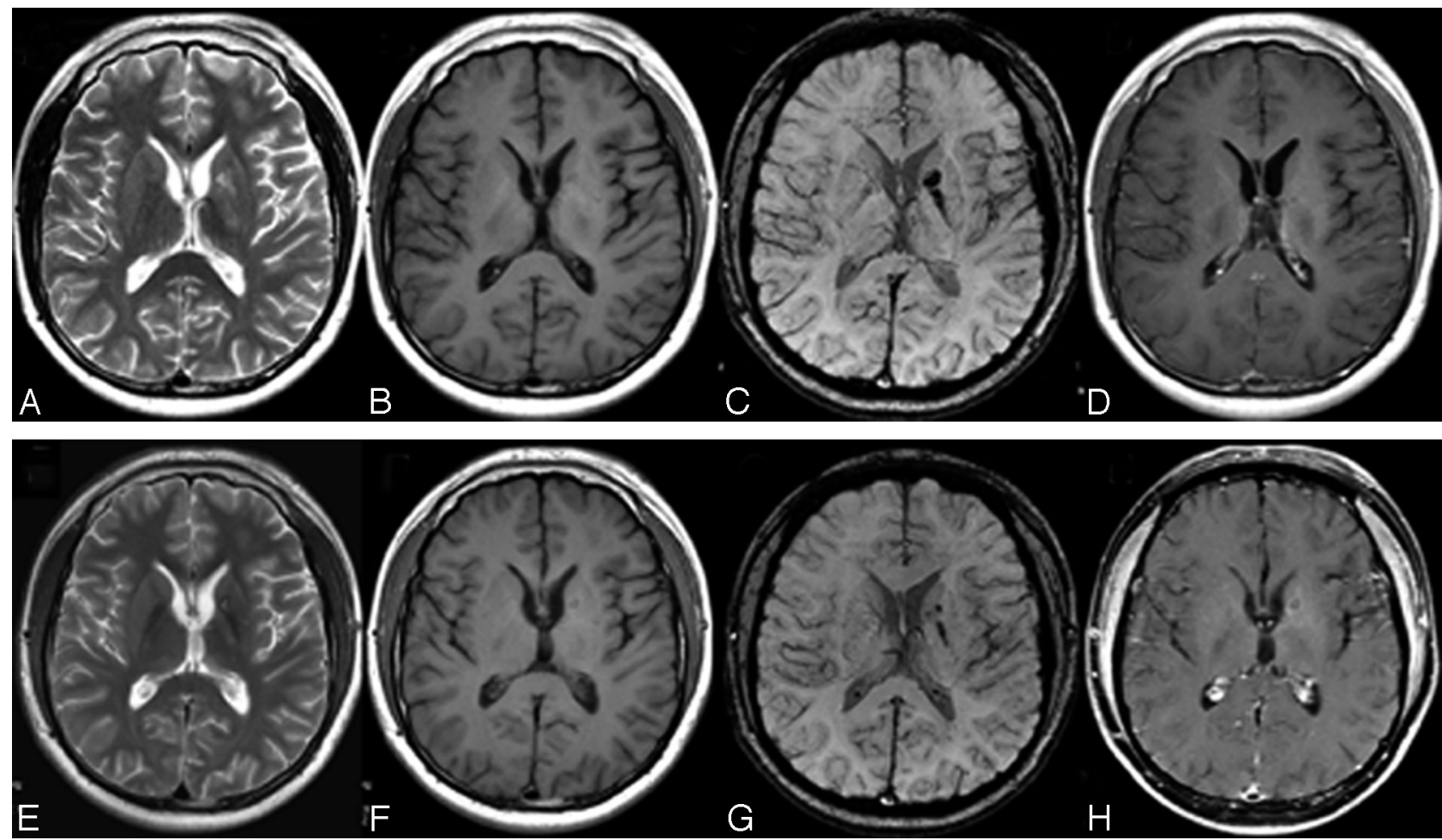

Fig 2. MR imaging of case 2. A, Axial T2WI shows patchy hyperintensity at the left BG. $B$, The tumor is invisible on T1WI. C, SWI shows obvious hypointensity at the globus pallidus, the genu, and the posterior limb of the internal capsule on the left side. D, Postcontrast T1WI shows no enhancement. $E-H$, Twenty-seven days after RT, the tumor markedly decreases on follow-up T2WI (E), T1WI (F) (still invisible), and SWI (G) but shows slight ringlike enhancement $(H)$.

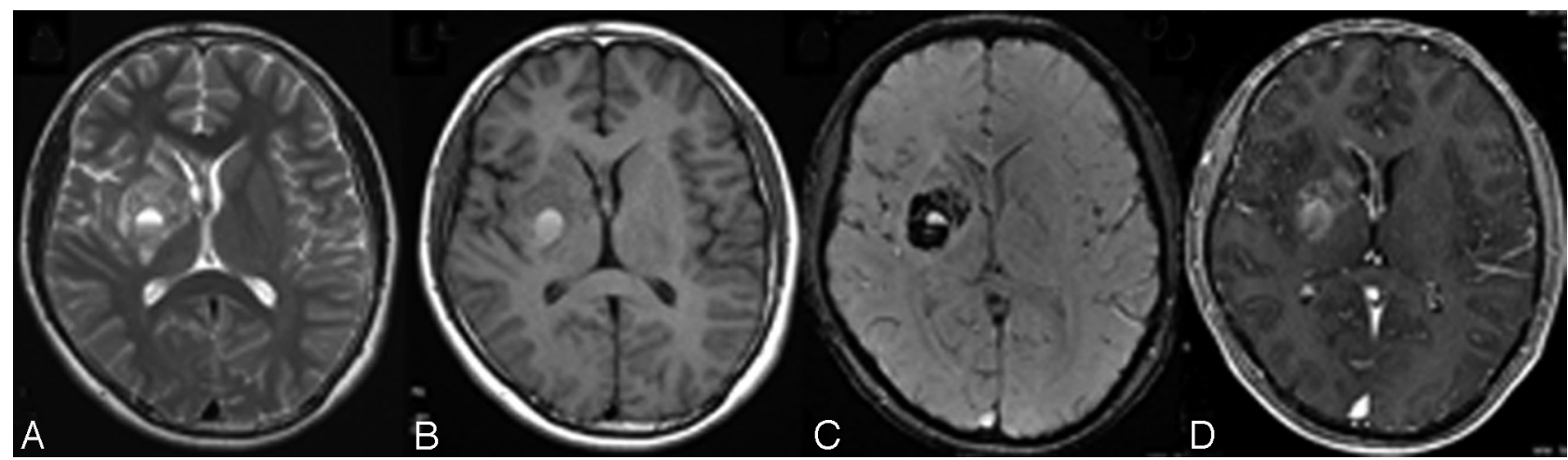

Fig 3. MR imaging of case 4. $A$ and $B$, Axial T2WI $(A)$ and T1WI $(B)$ show heterogeneous signal-intensity changes at the right $B G$, involving the genu of corpus callosum and septum pellucidum, and the right thalamus is also involved (not shown). C, SWI demonstrates obvious heterogeneous hypointensity together with fluid-fluid level. D, Heterogeneous enhancement in the $\mathrm{BG}$ and subependymal spread are noted on the postcontrast T1WI.

\begin{tabular}{lrrr}
\hline \multicolumn{4}{l}{ Table 3: Contrast-to-noise ratio for tumors on T1WI, T2WI, and SWI } \\
\hline Case & & & \\
No. & T1WI & T2WI & SWI \\
\hline 1 & 22.58 & 32.29 & -80.43 \\
2 & 13.30 & 67.48 & -100.29 \\
3 & 152.46 & 16.62 & -10.74 \\
4 & 25.60 & 130.10 & -15.90 \\
5 & -18.19 & 197.00 & -8.64 \\
6 & 18.94 & 45.13 & -70.98 \\
\hline
\end{tabular}

CNRs of tumor were higher on SWI than on T1WI and T2WI in cases 1, 2, and 6 (Table 3), indicating that SWI improved the signal-intensity contrast between tumor and adjacent brain tissue in the early stage of BG germinoma.

When we compared conventional MR imaging with the
SWI investigations in all our patients, the locations of the tumor remained unchanged and SWI did not reveal any new localization that conventional MR imaging did not show, though, indeed, the tumor appeared larger in the globus pallidus and putamen in cases with early BG germinomas on SWI.

The median follow-up period was 12 months, and no recurrence was noted in 3 early BG germinomas after RT or in 3 late $\mathrm{BG}$ germinomas after subtotal resection.

\section{Histologic and Immunohistochemical Findings}

In all patients, the neoplastic infiltration was composed of large round cells with moderate amounts of clear cytoplasm and prominent vesicular nuclei with plump nucleoli resembling primordial germ cells (Fig $4 A$, case 4 ). This predominant 

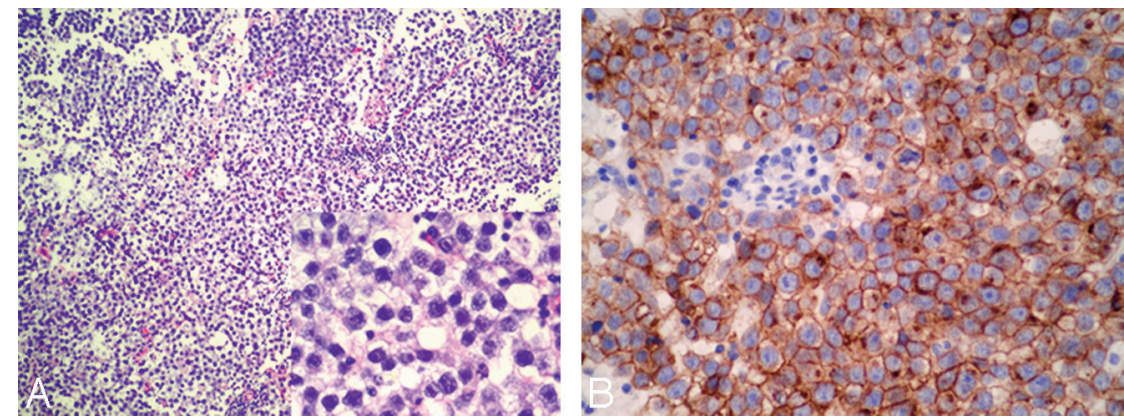

Fig 4. Photomicrographs of histopathology of the right BG germinoma in case 4 . $A$, Standard HE coloration (original magnification $\times 100$; insert figure: original magnification $\times 400$ ) shows that the tumor is composed of large cells with vacuolated cytoplasm and round or ovoid nuclei with prominent nucleoli. Lymphocytic element admixes with tumor cells focally. $B$, Immunolabeling of germ cells with CD117a (c-kit) is positive (original magnification $\times 400$ ).

\begin{tabular}{|c|c|c|c|c|c|c|c|}
\hline Reference & Total No. & Hemorrhage & $\begin{array}{l}\text { Mass } \\
\text { Effect }\end{array}$ & $\begin{array}{c}\text { Contrast } \\
\text { Enhancement }\end{array}$ & SWI & Radiologic Features on T1WI & $\begin{array}{c}\text { Radiologic Features } \\
\text { on T2WI }\end{array}$ \\
\hline Kim et al ${ }^{1}$ & 14 & 7 & 4 & 14 & None & Hyper in 7 , hetero in 7 & Hyper in 7 , hetero in 7 \\
\hline Klein et $\mathrm{al}^{2}$ & 1 & 0 & 1 & 1 & None & Hetero & Hetero \\
\hline Wong et al $\left.\right|^{3}$ & $\begin{array}{l}6 \text { (3 performed } \\
\text { MR imaging) }\end{array}$ & No data & 2 & 3 & None & Hetero in 3 & Hetero in 3 \\
\hline Moon et $\mathrm{al}^{5}$ & 10 & 7 & No data & 10 & None & Hyper in 5 , hetero in 5 & Hyper in 5 , hetero in 5 \\
\hline Higano et $\mathrm{al}^{6}$ & 6 & 1 & 3 & No data & None & hyper in 2 , hetero in 4 & Hyper in 2, hetero in 4 \\
\hline Liu et $\mathrm{al}^{7}$ & 1 & 0 & 0 & 1 & None & Нуро & Hetero \\
\hline Ozelame et $\mathrm{al}^{8}$ & 4 & 2 & 2 & 4 & None & Hyper in 2, hetero in 2 & Hyper in 2, hetero in 2 \\
\hline Rushing et al ${ }^{12}$ & 2 & 1 & 1 & No data & None & Hypo in 2 & Hyper in 1 , hetero in 1 \\
\hline Nagata et $\mathrm{al}^{13}$ & 1 & 0 & 0 & 1 & None & Нуро & Hyper \\
\hline Fujii et $a^{18}$ & 1 & 0 & 0 & 1 & Yes & Hyро & Hyper \\
\hline Current series & 6 & 6 & 3 & 3 & Yes & Iso in 2 , hyper in 1 , hetero in 3 & Hyper in 3 , hetero in 3 \\
\hline
\end{tabular}

germ cell component was intermingled with variable numbers of nonneoplastic lymphocytes, especially in the biopsy sample obtained in case 1 . What was most striking about the sample obtained in case 3 was that the infiltrate consisted mostly of individual and small collections of tumor cells that extensively permeated the cerebral cortex and subcortical white matter. Neoplastic germ cells could also be identified in perivascular and subpial collections in cases 4 and 5. Immunohistochemical staining showed expression of CD117a (c-kit) by the neoplastic cells in all cases (Fig 4B, case 4).

\section{Discussion}

Germinomas are commonly large and circumscribed lesions typically in the pineal or suprasellar regions, which infiltrate and compress adjacent structures causing various clinical symptoms. ${ }^{15}$ BG germinomas are rare, though their incidence in East Asia is higher. ${ }^{1,2}$ They have an absolute male predominance, most patients are younger than 20 years of age, and most BG germinomas progress slowly as seen in our study. ${ }^{1,2}$ Clinical manifestation of BG germinomas varies, including hemiparesis, mental deterioration, precocious puberty, diabetes insipidus, oculomotor palsy, speech disturbance, and hemianopia, etc. Signs of intracranial hypertension usually do not occur until the late stages.

Early recognition of BG germinoma is important because it is highly radiosensitive and potentially curable by RT alone if correctly diagnosed at its early stage. BG germinoma is one of the differential diagnoses when a lesion in the BG is ill-defined, hyperintense on T2WI, and without mass effect or prominent contrast enhancement in young patients. However, these changes are subtle and inconspicuous on conventional MR imaging. ${ }^{1}$ SWI exploits the magnetic-susceptibility effects generated by local inhomogeneity of the field. It can be used to detect blood products and biologic metal accumulation and provides additional information in brain tumor evaluation, ${ }^{16}$ which is valuable in BG germinoma due to its characteristic intratumoral hemorrhage. SWI acquired with a gradient recalled-echo sequence is more suitable than SWI with a spinecho sequence in evaluating tumor vascularity. ${ }^{17}$ The clinical data and MR imaging findings of BG germinoma in the literature are summarized in Table 4 , with only 1 previous article using the SWI technique. ${ }^{18}$ Hence, we aimed to evaluate and compare SWI with conventional MR imaging in the diagnosis of early BG germinomas.

For the purpose of our study, early BG germinomas are defined as tumor size $<10 \mathrm{~mm}$ in largest diameter without cystic changes, necrosis, or hemorrhage on conventional MR imaging. Three cases (cases 1, 2, and 6) were classified as an early subtype with patchy hyperintensity on T2WI without contrast enhancement. In our study, distinct patterns between early and late BG germinomas have emerged on the basis of the comprehensive findings on both conventional MR imaging and SWI sequences. Although early BG germinomas have some distinct characteristics, (ie, hyperintense T2 signal intensity and no enhancement), they are more strikingly visualized and appear larger on SWI sequences than on conventional MR imaging. For late BG germinomas, however, the overall tumor visibility and boundary definition were worse on SWI because it merely demonstrates intratumoral blood products and biologic metal deposition but not edema or subependymal tumor 
spread. As the tumor progresses and increases in size and complexity, it is better delineated by conventional MR images due to its more obvious signal-intensity heterogeneity on T1WI and T2WI as well as contrast uptake at the late stage.

In addition to intratumoral hemorrhage, signal-intensity change of germinomas on SWI may also be explained by 2 other mechanisms: First, BG structures, especially the internal capsule and globus pallidus, are rich in biologic metals such as iron. Tumor invasion may lead to disruption in axonal transport of transferrin, resulting in abnormal iron accumulation. Second, it is possible that transferrin secreted by the tumor cells binds to transferrin receptors on neurons and glial cells, which are then transported into the globus pallidus. ${ }^{16,19}$ Further histologic-radiologic correlation studies are needed to support the proposed mechanisms.

Diabetes insipidus was encountered in 2 patients (cases 2 and 5). No mass or abnormal enhancement was seen in the region of the hypothalamus or infundibulum on initial MR imaging, no new lesion was detected on follow-up MR imaging, and symptoms resolved after RT. We speculated that subtle coexisting tumor infiltration in the regions of the hypothalamus or infundibulum may be a reasonable explanation for the clinical manifestation, and MR imaging follow-up is crucial and highly recommended in such patients.

\section{Conclusions}

SWI is useful in the detection of early BG germinomas due to the presence of intratumoral blood products and biologic metal accumulation and has a distinctive radiologic pattern combined with conventional MR images, which adds important information in the early diagnosis of this potentially curable condition.

\section{References}

1. Kim DI, Yoon PH, Ryu YH, et al. MRI of germinomas arising from the basal ganglia and thalamus. Neuroradiology 1998;40:507-11
2. Klein $\mathrm{O}$, Voirin J, Civit $\mathrm{T}$, et al. Germinoma located in the basal ganglia in an 8-year-old girl. Childs Nerv Syst 2007;23:105-08

3. Wong TT, Chen YW, Guo WY, et al. Germinoma involving the basal ganglia in children. Childs Nerv Syst 2008;24:71-78

4. Komatsu Y, Narushima K, Kobayashi E, et al. CT and MR of germinoma in the basal ganglia. AJNR Am J Neuroradiol 1989;10(5 suppl):S9-11

5. Moon WK, Chang KH, Kim IO, et al. Germinomas of the basal ganglia and thalamus: MR findings and a comparison between MR and CT. AJR Am J Roentgenol 1994;162:1413-17

6. Higano S, Takahashi S, Ishii K, et al. Germinoma originating in the basal ganglia and thalamus: MR and CT evaluation. AJNR Am J Neuroradiol 1994;15:1435-41

7. Liu E, Robertson RL, du Plessis A, et al. Basal ganglia germinoma with progressive cerebral hemiatrophy. Pediatr Neurol 1999;20:312-14

8. Ozelame RV, Shroff M, Wood B, et al. Basal ganglia germinoma in children with associated ipsilateral cerebral and brain stem hemiatrophy. Pediatr Radiol 2006;36:325-30

9. Huh SJ, Shin $\mathrm{KH}$, Kim IH, et al. Radiotherapy of intracranial germinomas. Radiother Oncol 1996;38:19-23

10. Liang L, Korogi $Y$, Sugahara T, et al. Detection of intracranial hemorrhage with susceptibility-weighted MR sequences. AJNR Am J Neuroradiol 1999;20: 1527-34

11. Tong KA, Ashwal S, Holshouser BA, et al. Hemorrhagic shearing lesions in children and adolescents with posttraumatic diffuse axonal injury: improved detection and initial results. Radiology 2003;227:332-39

12. Rushing EJ, Sandberg GD, Judkins AR, et al. Germinoma: unusual imaging and pathological characteristics-report of two cases. J Neurosurg 2006;104: $143-48$

13. Nagata $\mathrm{K}$, Nikaido $\mathrm{Y}$, Yuasa $\mathrm{T}$, et al. Germinoma causing wallerian degeneration: case report and review of the literature. J Neurosurg 1998;88: $126-28$

14. Lee BC, Vo KD, Kido DK, et al. MR high-resolution blood oxygenation leveldependent venography of occult (low-flow) vascular lesions. AJNR Am J Neuroradiol 1999;20:1239-42

15. Sugimoto K, Nakahara I, Nishikawa M. Bilateral metachronous germinoma of the basal ganglia occurring long after total removal of a mature pineal teratoma: case report. Neurosurgery 2002;50:613-16

16. Sehgal V, Delproposto Z, Haddar D, et al. Susceptibility-weighted imaging to visualize blood products and improve tumor contrast in the study of brain masses. J Magn Reson Imaging 2006;24:41-51

17. Uematsu H, Matsuda T, Takahashi M, et al. Susceptibility-induced changes in signal intensity from spin-echo versus gradient-echo sequences. Clin Imaging 2002;26:367-70

18. Fujii $\mathrm{Y}$, Saito $\mathrm{Y}$, Ogawa $\mathrm{T}$, et al. Basal ganglia germinoma: diagnostic value of MR spectroscopy and (11)C-methionine positron emission tomography. J Neurol Sci 2008;270:189-93. Epub 2008 Mar 26

19. Rouault TA, Cooperman S. Brain iron metabolism. Semin Pediatr Neurol 2006; $13: 142-48$ 